\title{
COMMUNICATION
}

\section{Le prélèvement osseux autogène en site palatin : une étude radiologique de faisabilité}

\section{Delebarre $\mathrm{H}^{1}$, , Borie $\mathrm{G}^{1}$ 2, Paul $\mathrm{A}^{\mathbf{1}} \mathbf{2}$}

1 - CHU de Nice, Hôpital Saint-Roch, Pôle Odontologie, 5 rue Pierre Dévoluy, 06000 Nice, France

2 - UFR d'Odontologie de Nice, UNS, 24 avenue des Diables Bleus, 06000 Nice, France

\section{Introduction}

La pose d'implant est tributaire du volume osseux disponible. L'indication d'une greffe osseuse pré-implantaire se pose régulièrement face à un déficit osseux non compensable par une technique alternative. L'os autogène offre l'avantage d'être immuno-compatible en éliminant le risque de contamination, et associe 3 propriétés fondamentales : ostéoinduction, ostéoconduction et ostéogenèse, qui font de lui un greffon de référence. Les sites de prélèvements intra-oraux les plus utilisés correspondent à la tubérosité maxillaire, la zone rétro-molaire et ramique mandibulaire ainsi que la zone symphysaire paramédiane. Chacune de ces zones présentent des caractéristiques anatomiques à maîtriser lors de l'abord chirurgical. Le prélèvement osseux palatin rétro-incisif constitue à ce titre une approche intéressante. L'objectif de cette étude est de déterminer radiologiquement si le prélèvement osseux palatin est une technique réalisable et reproductible.

\section{Matériels et Méthodes}

L'analyse radiologique a été réalisée à partir de reconstructions tridimensionnelles de conebeam, réalisées au centre de soins dentaires sur des patients du CHU de Nice, entre 2014 et 2015. Ont été retenus les patients âgés de 20 à 60 ans, ne présentant pas de canine maxillaire incluse. Des mesures ont été réalisées afin de délimiter un volume de prélèvement suffisant, en respectant des marges de sécurité de $4 \mathrm{~mm}$ par rapport aux racines dentaires en regard du trait de coupe. Une première mesure caractérisait la hauteur d'os disponible au niveau de la deuxième prémolaire maxillaire, de la canine et du foramen incisif jusqu'au plancher des fosses nasales, puis une seconde mesure antéro-postérieure représentait la longueur entre le foramen incisif et la ligne reliant les deuxièmes prémolaires maxillaires. Ces mesures formant un volume tridimensionnel disponible au prélèvement du bloc osseux. Résultats : 100 patients ont été inclus dans cette étude radiologique, avec un sex-ratio équilibré (53 femmes et 47 hommes). L'âge moyen était de 47,5 ans. La moyenne du volume osseux disponible dans cette zone anatomique était relativement variable, avec une longueur de 15,61 mm entre le foramen incisif et son intersection avec la ligne joignant les deuxièmes prémolaires maxillaires, une hauteur d'os de $14,38 \mathrm{~mm}$ au niveau de la canine jusqu'au plancher des fosses nasales, ainsi qu'une hauteur de 10,84 mm au niveau de la deuxième prémolaire, en respectant une marge de sécurité de $2 \mathrm{~mm}$ par rapport aux structures anatomiques sus-jacentes (fosses nasales et sinus maxillaires). 
Discussion

La greffe osseuse alourdit le traitement implantaire cependant elle est souvent incontournable. Le prélèvement intrabuccal, par sa structure et nature biologique, offre un matériau quasiment identique à son substrat. La greffe osseuse autologue palatine constitue une voie intéressante par sa situation anatomique à faible risque, sa moindre morbidité, et le volume osseux disponible tant cortical que spongieux. L'abord chirurgical nécessite quant à lui un décollement étendu de la muqueuse palatine. Même si des variations dimensionnelles inter-individuelles existent, l'approche de ce site de prélèvement prend tout son sens dans des reconstructions préimplantaires de faible étendue et plus particulièrement pour des techniques de greffes par coffrage décrites, entre autres, par le Pr F. Khoury.

Conclusion

Le prélèvement osseux autogène en site palatin semble être, d'après les résultats de cette étude, une technique réalisable et reproductible pour des greffes osseuses de faible étendue. Une étude de faisabilité sur cadavre est prévue, suivie éventuellement d'une série de cas cliniques. 